\title{
F-DICE: A Multiple Node Upset Tolerant Flip- Flop for Highly Radioactive Environments
}

\author{
Stefano Campitelli, \\ Marco Ottavi, \\ Salvatore Pontarelli \\ Electronic Engineering Department \\ University of Rome "Tor Vergata" \\ Rome, Italy \\ stefano.campitelli@gmail.com, \\ \{ottavi,pontarelli\}@ing.uniroma2.it
}

\author{
Alessandro Marchioro, \\ Daniele Felici \\ CERN European Organization \\ for Nuclear Research \\ Department: PH Group: ESE \\ 1211 Geneve 23, Switzerland \\ alessandro.marchioro@cern.ch, \\ daniele.felici@cern.ch
}

\author{
Fabrizio Lombardi \\ Department of Electrical \\ and Computer Engineering \\ Northeastern University \\ Boston, MA, USA \\ lombardi@ece.neu.edu
}

\begin{abstract}
This paper introduces a novel design for a multiple node upset tolerant flip-flop. This design uses the TDICE memory cell that was proposed in the technical literature for memory arrays and applies its principles of operation to a Master Slave flip-flop implemented at $65 \mathrm{~nm}$ CMOS technology. It is shown that the proposed design approach is particularly suited for flip-flops targeting highly radioactive environments; simulation validates the multiple node upset tolerance and its viability. A test chip developed for the on-silicon validation is also described.
\end{abstract}

Keywords-Multiple node upset, Flip flop, radiation

\section{INTRODUCTION}

With the constant scaling of transistors' feature size, one of the most important reliability problems affecting the semiconductor industry in recent years is the interaction with ionized particles [1] and to atmospheric neutrons [2]. These interactions can cause glitches in combinatorial logic and a permanent loss of information in memory elements when the so-called Single Event Upset (SEU) occurs; a SEU flips the value stored in a memory element.

Different approaches have been proposed to tolerate the presence of SEUs; fault avoidance approaches (such as found in [3] [4]) are targeted at modifying the technology or the design of the memory cells (examples can be found in [5], [6].) to make them tolerant to the presence of injected charge and consequently avoiding the occurrence of the bit flip. Fault tolerant approaches instead target at tolerating the occurrence of the bit flips at system level by adding different redundancy approaches such as coding (information redundancy) TMR/DMR (hardware redundancy) repeated operations (time redundancy) [7]. Depending on the size and the use of the memory to be protected (register files, cache memories, main memories, storage memories), different ECCs have been used [8], [9], [10], [11]. However, these techniques are not suitable for the protection of single memory elements such as latches or flip-flops.

For these cases circuit-level fault tolerance techniques are more efficient. Many circuit-level fault avoidance approaches have been proposed in the past to make the circuit of memory cells tolerant to the presence of charge injection at any of their nodes [5] [6]. The DICE cell [12] is one of the most popular designs that over the years has become nearly a standard for SEU tolerant circuit design. However the DICE-cell as well as other SEU tolerant designs are now facing new challenges caused by the so-called charge sharing effect. As device size shrinks, spacing between nodes decreases significantly and the charge generated from a single event strike may diffuse, thus affecting adjacent nodes, i.e. charge sharing occurs [13]. The adjacency can be considered at circuit-level too. As a single event occurs, the multiple node upset scenario is likely to require new designs for hardening memory cells. The occurrence of SEU with multiple node upset also more likely when a high rate of impacts from extremely high-energy particles is expected. This is the case of the Large Hadron Collider at CERN, where part of this work was carried out. The flip-flop presented in this work has been designed to work in that radiation environment that is at least ten times more radioactive than the space environment. Usually circuitlevel hardware redundancy techniques are also used to protect the flip-flops from a SEU [14] [15] . While memory arrays use efficient error detection and correction codes, the sparse and irregular placement of flip-flops into a chip, makes it less effective when applying techniques based on error codes.

The objective of this paper is to introduce a novel flip-flop design based on a previously introduced Multiple Node Upset Tolerant cell known as TDICE [16]. This design leverages the principle of operation of the TDICE cell by adapting it to a master slave flip flop and in particular, by taking into account the specific design constraints of a high Total Ionizing Dose (TID) environment present in experiments carried out at CERN.

\section{BACKGROUND}

This section shows a brief review of soft error modeling and the techniques for protecting information from a SEU.

\section{A. Single Event Upset Modeling}

When an alpha particle strikes silicon, it travels and loses energy along a straight path[3]. The denser the material is, the quicker a heavy ionizing particles loses its energy because there is a higher density of charge with which to interact. As heavy ionizing particles loses its kinetic energy (thus reducing 
its velocity), it allows more time for its positive charge to induce electron-hole pairs through Coulombic interaction. Consequently, the charge generation rate increases with the distance traveled by the heavy ionizing particles and reaches a maximum near the end of the heavy ionizing particles path. The phenomenon of a heavy ionizing particles striking MOS devices has been extensively studied; it is modeled by a time varying double-exponential current pulse given by :

$$
I(t)=I_{0}\left(e^{-\frac{t}{\tau_{\alpha}}}-e^{-\frac{t}{\tau_{\beta}}}\right)
$$

where $\tau_{\alpha}$ is the collecting time constant of the junction and $\tau_{\beta}$ is the time constant for initially establishing the ion track. The parameter $I_{0}$ depends on the amount of injected charge. The time constants for the exponentials depend on several process factors [17]. From the current expression, it is possible to calculate the associated critical charge by integrating it, i.e.

$$
Q(t)=I_{0} \int\left(e^{-\frac{t}{\tau_{\alpha}}}-e^{-\frac{t}{\tau_{\beta}}}\right)
$$

If the collected charge reaches a critical value that is sufficiently high to result in a voltage value over the fanout gate logic threshold, an incorrect value can be propagated. Therefore, the heavy ionizing particle hit has been simulated by connecting an ideal current generator to one or more affected nodes [6] [12]. The affected MOS is replaced by a current generator as per the current equation shown in figure 1; this simulation-based approach has been verified to yield the same correct results as a 3D device modeling approach [18].

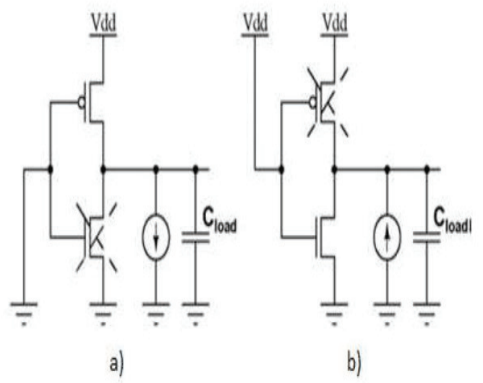

Fig. 1. Simulation Model

\section{B. DICE based Flip-Flop}

Regular arrays made of memories allow a designer to use high efficient error detection and correction codes, such as orthogonal codes, flip-flops are usually placed in a sparse and irregular manner on a chip, and therefore it is not possible to easily apply techniques based on error codes. Therefore, hardware redundancy techniques are usually exploited to protect the flip-flops from a SEU. In a DICE flip-flop the redundancy plays an essential role to protect the stored data from a SEU.

A DICE latch has a 4-nodes redundant structure; this implies that the logic state of each of the four nodes of the cell is controlled by two adjacent nodes located on the opposite diagonal. The schematic diagram of the DICE latch

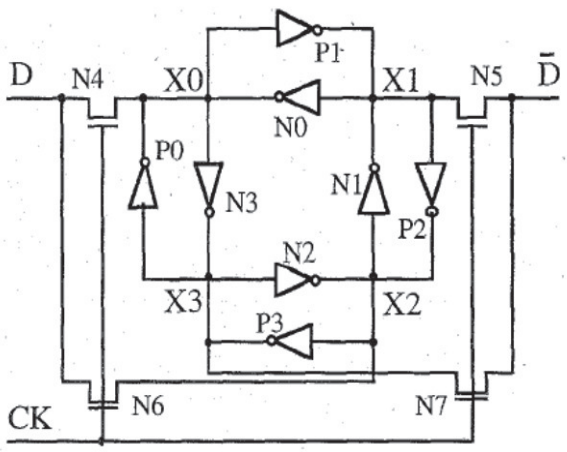

Fig. 2. DICE Architecture

is presented in figure 2. The DICE latch is fully tolerant to a single event affecting only one node, but it is sensitive to a multiple node upset. The multiple node upset happens when a single event effect influences at least two different nodes and both of them change their state.

\section{PROPOSED DESIGN}

In this section, the proposed flip-flop design for multiple node upset tolerance is presented; this new design is referred to as F-DICE. A typical DICE cell is protected from any single node upset, but it has little protection from a multiple node upset. The basic principle of the proposed design is to insert four NMOS transistors to block the feedback on the PMOS gate of a DICE cell. If a particle strikes two sensitive nodes, the block transistors protects the charge stored in the PMOS gate capacity and cause an increase of the critical charge of the cell at the sensitive nodes. Figure 3 shows the proposed design arranged in a typical master/slave structure, the master and the slave latches are the same. The single latch has two working modes:

- Writing mode : in this mode, the output of the latch follows its input. There is no protection from a multiple bit upset, because the block transistors are closed.

- Holding mode : in this mode, the latch holds the output fixed to the stored logic value. Block transitors disconnect the PMOS gate to prevent the error to propagate to adjacent nodes.

The inverted clock, clearly, drives the slave latch. When the master latch is in holding mode, the slave latch is in writing mode and vice versa. Figure 3 shows that the master latch NMOS block transistors (N3, N4, N5, N6) are driven by the inverted clock; the write operation of the latch is done by changing the logical state of two nodes. This change is done through two transmission gates (TG1, TG2). To simplify the writing operation, there are two NMOS transistors (N1, N2) that block the feedback when TG1 and TG2 try to change the state of the FF. In this cell there is also a chain of two inverters for the generation and the propagation of the clock and inverted clock signals. The proposed flip flop design was implemented by full custom flow and targeted to a test chip developed and taped out at CERN using TSMC 65nm technology.

The size of the cell is $1.8 \mu \mathrm{m} \times 15.2 \mu \mathrm{m}$. The height of the cell is the same of a standard cell in TSMC library. The 
average distance between two sensitive nodes is about $1.5 \mu \mathrm{m}$. To equalize the clock delay of the master and slave latches the clock driver is located in the middle of the cell. For the routing of the signal inside the cell is used two metal layer.

Furthermore, the developed flip flop is specifically targeted to highly radioactive environments typical of the experiments carried out at the CERN Large Hadron Collider where the total dose is expected to be as high as $100 \mathrm{MRad} / 10$ years.

Due to the high quantities of radiation that the flip-flop must be able to tolerate, the flip-flop requires also the adoption of specific design techniques as countermeasures against the effects of total ionizing dose effect. In particular, the flip-flop has been designed with channel size of transistors of $300 \mathrm{~nm}$ [19]. As shown in Figure 4, the increase of leakage current caused by the total ionizing dose for devices with a channel width over $300 \mathrm{~nm}$ is less than ten times. accumulated ionizing dose.

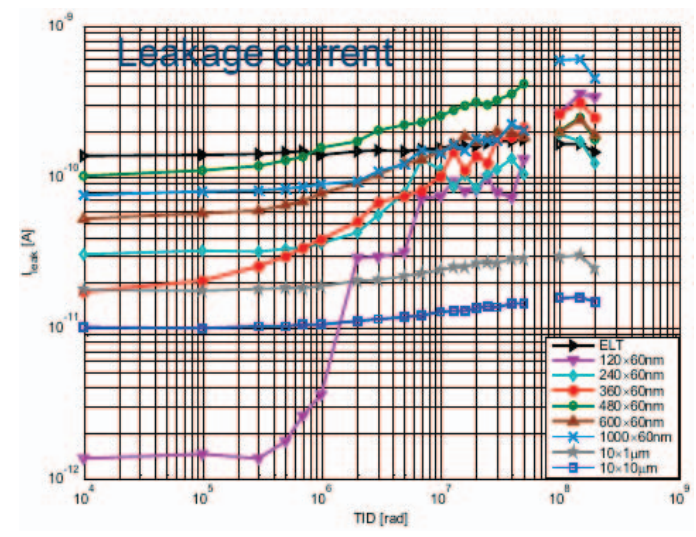

Fig. 4. TID Effect on NMOS in $65 \mathrm{~nm}$ technology

The use of wider transistors allows designing circuits that present a limited sensitivity to the TID effect, thus providing performances with limited time variability with respect to the accumulated ionizing dose.

\section{Simulation}

This section shows the multiple node-upset tolerance of the latch made of the designed flip-flops compared to its DICE counterpart implemented at the same $65 \mathrm{~nm}$ technology node. This simulation was performed using the SPECTRE simulator and the TSMC's model transistors. The simulated circuits are shown in figure 5

To validate the tolerance to multiple node upsets, the simulation was performed for every pair combination of circuit nodes affected by charge injection. The tolerance of the sensitive pairs in the TDICE latch increases of about $150 \%$ compared to the critical charge of the DICE cell in the same pairs. In some pairs the critical charge increase over $250 \%$.

As an example, figure 6 and 7 show the charge injected in $X_{0}$ and $X_{2}$ for a DICE and a TDICE latch. Every point in the figure is a simulation with different injected charge. The red area denotes a data corruption. The critical charges are different for pairs and nodes configurations. In this example, for 1-0-1-0 configuration, the critical charge is $150 \mathrm{fC}$ for a DICE latch and more than $300 \mathrm{fC}$ for a TDICE latch.
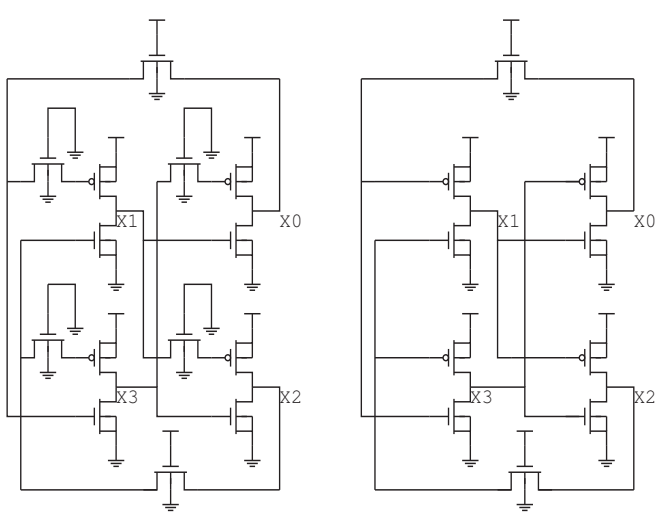

Fig. 5. Critical Charge Simulation

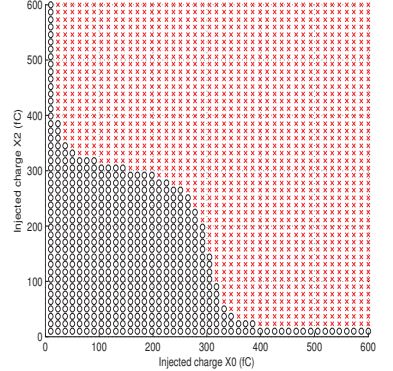

(a) Configuration Nodes : 0-1-0-1

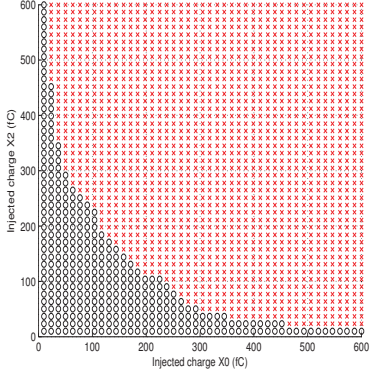

(b) Configuration Nodes : 1-0-1-0
Fig. 6. FF-DICE Critical Charge $X_{0}-X_{2}$

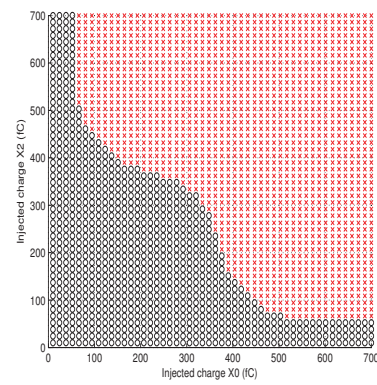

(a) Configuration Nodes : 0-1-0-1

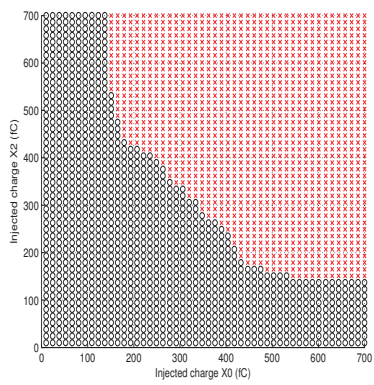

(b) Configuration Nodes : 1-0-1-0
Fig. 7. F-DICE Critical Charge $X_{0}-X_{2}$

\section{PERFormance EVAluation}

This section shows different figures of merit as related to performance metrics for flip-flop design; the following results were obtained through post-layout simulations.

\section{A. Timing and Delay}

The timing characteristics of the two designed flip-flops are reported on tables I and II for different process corner conditions; these tables show that the insertion of the four block transistors causes a loss of maximum clock speed of nearly $35 \%$. 


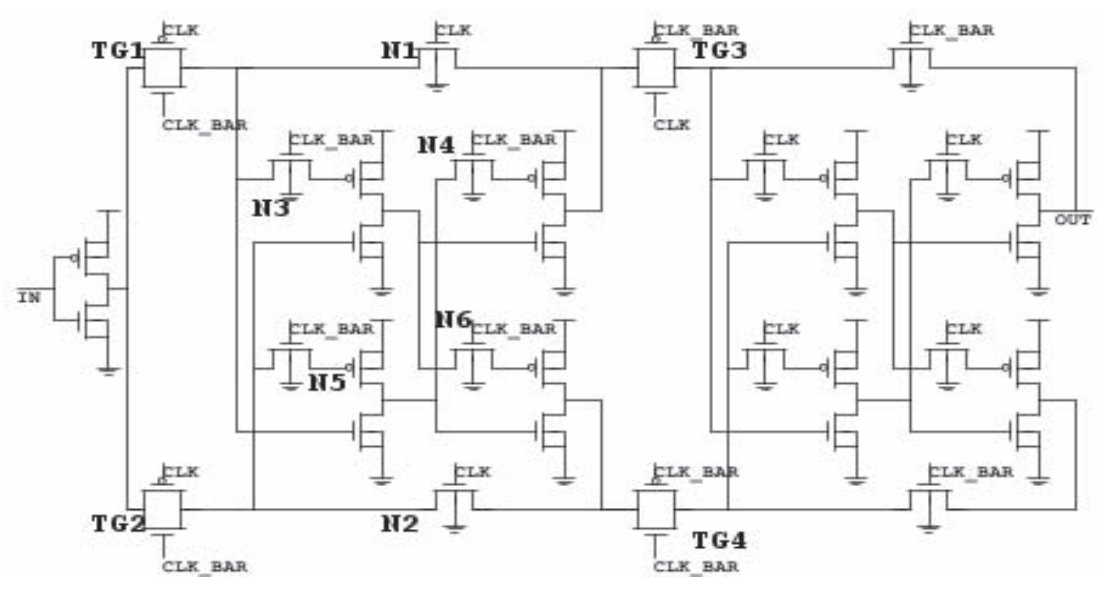

Fig. 3. F-DICE

TABLE I. FF-DICE TIMING

\begin{tabular}{|c|c|c|c|c|c|c|}
\hline \multirow{2}{*}{ FF-DICE } & \multicolumn{2}{|c|}{ Worst Case } & \multicolumn{2}{c|}{ Typical Case } & \multicolumn{2}{c|}{ Best Case } \\
\cline { 2 - 7 } & HL & LH & HL & LH & HL & LH \\
\hline Setup time & $112 p s$ & $93 p s$ & $60 p s$ & $45 p s$ & $35 p s$ & $28 p s$ \\
\hline Hold time & $36 p s$ & $54 p s$ & $27 p s$ & $46 p s$ & $11 p s$ & $32 p s$ \\
\hline Delay time & $227 p s$ & $247 p s$ & $128 p s$ & $142 p s$ & $79 p s$ & $88 p s$ \\
\hline
\end{tabular}

TABLE II. F-DICE TIMING

\begin{tabular}{|c|c|c|c|c|c|c|}
\hline \multirow{2}{*}{ F-DICE } & \multicolumn{2}{|c|}{ Worst Case } & \multicolumn{2}{c|}{ Typical Case } & \multicolumn{2}{c|}{ Best Case } \\
\cline { 2 - 7 } & HL & LH & HL & LH & HL & LH \\
\hline Setup time & $265 p s$ & $89 p s$ & $141 p s$ & $65 p s$ & $109 p s$ & $51 p s$ \\
\hline Hold time & $63 p s$ & $73 p s$ & $42 p s$ & $26 p s$ & $25 p s$ & $6 p s$ \\
\hline Delay time & $243 p s$ & $338 p s$ & $151 p s$ & $214 p s$ & $114 p s$ & $139 p s$ \\
\hline
\end{tabular}

\section{B. Area and Power Consumption}

The area of a single F-DICE is $1.8 \times 15.2 \mu \mathrm{m}^{2}$, while the area of a FF-DICE is $1.8 \times 11.4 \mu \mathrm{m}^{2}$. The insertion of the eight NMOS transistors (four in the master latch and four in the slave latch) increases the area of the F-DICE by nearly $30 \%$. The power consumption of the two designs is shown in tables III and IV.

TABLE III. CURRENT CONSUMPTION FF-DiCE

\begin{tabular}{|c|c|c|c|}
\hline FF-DICE @ $1 \mathrm{GHz}$ & Worst Case & Typical Case & Best Case \\
\hline$I_{\text {avg }}$ & $10.8 \mu \mathrm{A}$ & $12.8 \mu \mathrm{A}$ & $15.6 \mu \mathrm{A}$ \\
\hline$I_{\text {peak }}$ & $80 \mu \mathrm{A}$ & $155 \mu \mathrm{A}$ & $278 \mu \mathrm{A}$ \\
\hline
\end{tabular}

TABLE IV. CURRENT CONSUMPTiOn F-DiCE

\begin{tabular}{|c|c|c|c|}
\hline F-DICE @ $1 \mathrm{GHz}$ & Worst Case & Typical Case & Best Case \\
\hline$I_{\text {avg }}$ & $17.9 \mu \mathrm{A}$ & $19.3 \mu \mathrm{A}$ & $22.2 \mu \mathrm{A}$ \\
\hline$I_{\text {peak }}$ & $180 \mu \mathrm{A}$ & $221 \mu \mathrm{A}$ & $260 \mu \mathrm{A}$ \\
\hline
\end{tabular}

Tables III and IV are calculated at $1 G H z$ clock frequency. In this case, the F-DICE shows an overhead with respect to the FF-DICE of nearly $50 \%$

\section{Full Custom Test Chip Implementation}

To validate the proposed flip flop design, the Micro Electronics section at CERN also developed and taped out a test chip that fully passed the post silicon verification phase.

The test chip was used for a series of tests under radiation to confirm the simulation results on the effectiveness of the designed cell with respect to multiple node upset tolerance. To compare the effectiveness of the proposed design with respect to previous designs, the test chip implements standard flip flops, FF-DICE and the proposed F-DICE. Therefore, the test chip was composed of the following three shift registers:

1) The first shift register was composed of a chain of 7200 F-DICE.

2) The second shift register was composed of a chain of 7200 FF-DICE.

3) The third shift register was composed of a chain of 7200 standard cell flip-flops taken from the TSMC library.

The shift registers are organized in rows and columns, they are loaded from the same input, while they have three different output pads (see Figure 7). The size of the test chip was about $1 \mathrm{~mm} \times 1 \mathrm{~mm}$. At the beginning of every row there was a clock driver. The clock driver was composed of two stages driving $200 \mathrm{fF}$ for each row. The input of the clock driver was linked to the output of the previous clock driver. So the clock signal of every row was delayed from the previous one.

The reasons for the use of the clock skewing are :

- The die is bonded with golden wires of about 2 millimeters. They produce a not negligible parasitic inductance between pads and power that could be cause the simultaneous switching noise.

- The available area for power pads is limited.

The clock skewing allows to decrease the simultaneous switching noise and potential $d I / d t$ effects. The clock skew between two adjacent rows is about 120ps and it is obtained from the chain oh the two inverter. This skew makes sure that the current consumption is as constant as possible and consequently it makes sure that $d I / d t$ is the smallest possible.

\section{CONCLUSION}

The use of circuit level fault avoidance is an attractive solution in particular for flip-flops and register files that cannot exploit ECC codes with the same effectiveness of the intrinsically regular memory arrays. Moreover the constant shrinking of transistors feature sizes is increasingly jeopardizing the 


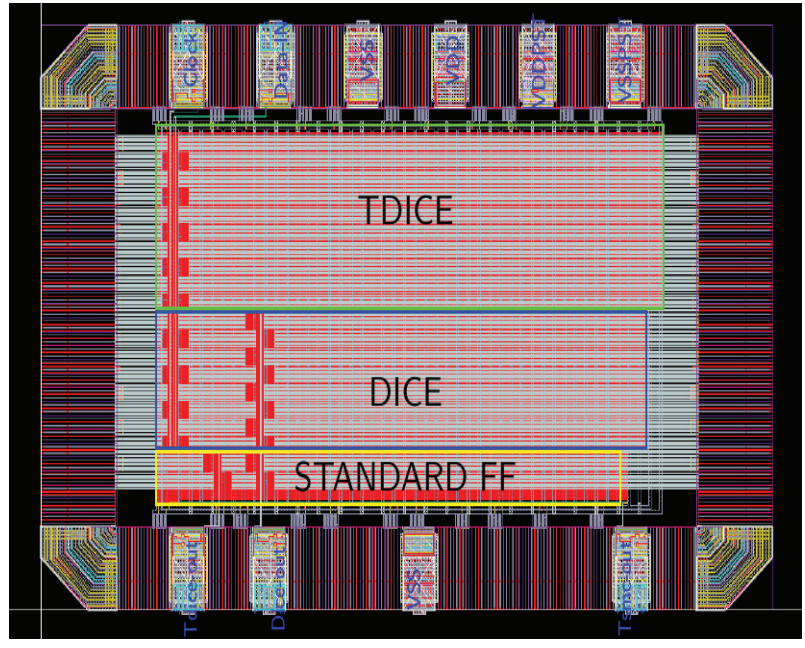

Fig. 8. Test Chip Layout

effectiveness of previous SEU tolerant circuit design due to the so-called charge sharing effect. Based on this motivation, this paper has proposed F-DICE a novel design of a flip-flop for improved SEU tolerance. The proposed design improves the critical charge of the sensitive nodes for multiple nodes upsets.

Furthermore F-DICE uses also specific hardening techniques conceived to be effective in highly radioactive environments, in particular for the total ionizing dose effects (such as those affecting the experiments at CERN). F-DICE has been verified through extensive simulation and it has also been implemented on a test chip that has been submitted to radiation experiments.

\section{ACKNOWLEDGMENT}

The authors would like to thank Marco D'Alessio for fruitful discussions and helpful comments.

\section{REFERENCES}

[1] R. C. Baumann, Radiation-induced soft errors in advanced semiconductor technologies, IEEE Transaction on Device and Materials Reliability, vol. 5 no. 3 pp. 305-316, 2005.

[2] M. Violante et al., A new hardware/software platform and a new 1/E neutron source for soft error studies: Testing FPGAs at the ISIS facility, IEEE Transaction on Nuclear Science, vol 54 no. 4 pp. 1184-1189, 2007.
[3] S. W. Fu, A. M. Mohsen and T. C. May, Alpha-particle-induced charge collection measurements and the effectiveness of a novel p-well protection barrier on VLSI memories IEEE Transaction Electron Devices. vol. 32 no. 1 pp. 49-54, 1985.

[4] J. D. Hayden, A quadruple well, quadruple polysilicon BiCMOS process for fast $16 \mathrm{Mb} S R A M$, IEEE Transaction Electron Devices, vol. 41 no. 12 pp.2318-2325, 1994.

[5] P. Hazucha, Measurement and analysis of SER-tolerant latch in $90 \mathrm{~nm}$ dual-VT CMOS process IEEE J. Solid-State Circuits, vol. 39 no. 9 pp. 1536-1543, 2004.

[6] S. Lin, Y. B. Kim and F. Lombardi, Soft-Error Hardening Designs of Nanoscale CMOS Latches, in Proc. IEEE VTS 2009, pp. 41-46, 2009.

[7] S. Mukherjee, Architecture Design for Soft Errors, Elsevier/Morgan Kaufmann, 2011.

[8] P. Reviriego, S. Pontarelli, J. A. Maestro, M. Ottavi, A Method to Construct Low Delay Single Error Correction Codes for Protecting Data Bits Only, Computer-Aided Design of Integrated Circuits and Systems, IEEE Transactions on vol. 32 no. 3, pp. 479-483, 2013.

[9] C. W. Slayman, Cache and memory error detection, correction, and reduction techniques for terrestrial servers and workstations, Device and Materials Reliability, IEEE Trans. on, vol. 5 no. 3, pp. 397-404, 2005.

[10] G. C. Cardarilli, M. Ottavi, S. Pontarelli, M. Re, A. Salsano, Fault tolerant solid state mass memory for space applications, Aerospace and Electronic Systems, IEEE Trans. on, vol. 41, no. 4, pp. 1353-1372, 2005.

[11] G. C. Cardarilli, M. Ottavi, S. Pontarelli, M. Re, A. Salsano Data integrity evaluations of Reed Solomon codes for storage systems, in Proc. of 19th IEEE Int. Symp. on Defect and Fault Tolerance in VLSI Systems, 2004. DFT 2004, pp.158-164.

[12] T. Calin, M. Nicolaidis, R. Velazco, Upset Hardened Memory Design for Submicron CMOS Technology, IEEE Transactions on Nuclear Science, vol. 43 no. 6 pp. 2874-2878, 1996.

[13] O. A. Amusan, A. F. Witulski, L. W. Massengil, B. L. Bhuva, P. R. Fleming, M. L. Alles, A. L. Sternberg, J. D. Black, R. D. Schrimpf, Charge collection and charge sharing in a 130nm CMOS Tecnology, IEEE Transactions on Nuclear Science, vol. 53, no. 6, pp. 3253-3258, 2006.

[14] M. Omana, D. Rossi, C. Metra, High-Performance Robust Latches, IEEE Transactions on Computers, vol. 59 no. 11, pp. 1455-1465, 2010.

[15] Y. Lu, F. Lombardi, S. Pontarelli, M. Ottavi, On the design of two single event tolerant slave latches for scan delay testing, in Proc. of IEEE Int. Symp. on Defect and Fault Tolerance in VLSI and Nanotechnology Systems (DFT12) pp. 67-72, 2012.

[16] M. D'Alessio, M. Ottavi, F. Lombardi, Design of a Nanometric CMOS Memory Cell for Hardening to a Single Event with a Multiple Node Upset,IEEE Transactions on Device and Materials Reliability, 2012.

[17] F. L. Yang and R. A. Saleh, Simulation and Analysis of Transient Faults in Digital Circuits, IEEE J. Solid State Circuits, vol. 27, no. 3, pp. 258264, 1992.

[18] G. C. Messenger, Collection of Charge on Junction Nodes from Ion Tracks, IEEE Transactions on Nuclear Science, vol. 29, no. 6, pp. 20242031, 1982.

[19] S. Bonacini, P. Valerio, Characterization of a commercial $65 \mathrm{~nm}$ CMOS technology for SLHC applications, Journal of Instrumentation, vol.7 no.1, 2012. 